\title{
Relato de experiência sobre uma intervenção psicológica com familiares de praticantes de equoterapia
}

\section{Experience report on a psychological intervention with family members of hippotherapy practitioners}

\section{Relato de experiencia acerca de una intervención psicológica con familiares de practicantes de equinoterapia}

Recebido: $29 / 08 / 2020$

Aprovado: 01/11/2020

Publicado: 09/01/2021
Sabrina Martins Barroso ${ }^{1}$ Juliana Machado Ruiz ${ }^{2}$ Camila Aparecida Peres Borges ${ }^{3}$ Cleyton Magela Reis ${ }^{4}$ Dennis Gabiatti Lopes ${ }^{5}$

O objetivo desse trabalho é relatar a experiência de um projeto de extensão baseado em intervenção psicológica breve realizado com cuidadores de praticantes de equoterapia. 0 projeto ocorreu como uma extensão universitária ao longo de 2018, e contou com 19 familiares cuidadores. Inicialmente, avaliou-se o estado emocional e a rotina dos cuidadores. Com base no relato dos familiares, definiuse o foco da intervenção. Foram organizados nove encontros individuais ou com casais de cuidadores, nos quais abordou-se a rotina de cuidado, atividades de lazer, divisão de tarefas e autocuidado. Percebeu-se que intervenções focadas nos cuidadores podem contribuir para melhorar a qualidade dos cuidados prestados aos usuários de equoterapia e geram impacto positivo em sua qualidade de vida, representando uma medida de promoção da saúde.

Descritores: Cuidadores; Relações Comunidade-Instituição; Psicologia; Relatos de casos.

This study aims to report the experience of an extension project based on a brief psychological intervention carried out with caregivers of horse riding practitioners. The project took place as a university extension, throughout 2018, and it consisted of 19 family caregivers. Initially, the caregivers' emotional state and routine were assessed. The focus of the intervention was defined based on the report of family members. Nine individual meetings or with couples of caregivers were organized, in which the routine of care, leisure activities, division of tasks and self-care were all addressed. It was possible to notice that interventions focused on caregivers can contribute to the improvement of the quality of care they provide to users of hippotherapy, and it can also have a positive impact on their quality of life. Therefore, it represents a health promotion measure.

Descriptors: Caregivers; Community-Institutional Relations; Psychology; Case reports.

El objetivo de este trabajo es relatar la experiencia de un proyecto de extensión basado en una breve intervención psicológica llevada a cabo con los cuidadores de los practicantes de equinoterapia. El proyecto se llevó a cabo como una extensión universitaria durante todo el año 2018 y contó con 19 familiares cuidadores. Inicialmente, se evaluó el estado emocional y la rutina de los cuidadores. Basándose en el relato de los familiares, se definió el foco de la intervención. Se organizaron nueve reuniones individuales o con parejas de cuidadores, en las que se trató la rutina de los cuidados, las actividades de ocio, la división de tareas y el autocuidado. Se observó que las intervenciones centradas en los cuidadores pueden contribuir a mejorar la calidad de los cuidados que prestan a los usuarios de la equinoterapia y generar un efecto positivo en su calidad de vida, lo que representa una medida de promoción de la salud.

Descriptores: Cuidadores; Relaciones Comunidad-Institución; Psicología; Informes de casos.

1. Psicóloga. Especialista em Avaliação Psicológica e Desenvolvimento Humano. Mestre em Psicologia. Doutora em Saúde Pública. Professora Adjunta e coordenadora do Programa de Pós Graduação em Psicologia (PPGP) da Universidade Federal do Triângulo Mineiro (UFTM), Uberaba, MG, Brasil. ORCID: 0000-0003-1759-9681 E-mail: smb.uftm@gmail.com

2. Psicóloga. Especialista em Psicologia Hospitalar e da Saúde. Mestre em Psicologia. Especializanda em Reabilitação Física na modalidade Residência Multiprofissional pela Faculdade de Medicina de São José do Rio Preto, São José do Rio Preto, SP, Brasil. ORCID: 0000-0002-0895-5253. E-mail: julianamruiz@hotmail.com

3. Psicóloga. Especialista em Psicologia Jurídica. Mestre em Psicologia. Psicóloga na Clínica Humanamente. Uberaba, MG, Brasil. ORCID: 0000-0001-7419-8919. E-mail: camilaappborges@gmail.com

4. Psicólogo. Associação Mineira de Equoterapia, Uberaba, MG, Brasil. ORCID: 0000-0002-5956-0083 E-mail: equoterapia.ame@yahoo.com

5. Psicólogo. Especialista em Análise do Comportamento Aplicada ao Autismo, UFSCAR, São Carlos, SP. ORCID: 0000-0003-3721-9922

E-mail: dglp12@hotmail.com 


\section{INTRODUÇÃO}

A

Equoterapia é uma técnica reconhecida por agir na recuperação de danos motores, sensoriais, cognitivos e comportamentais, por meio de equitação, medidas reeducativas

e lúdico-esportivas ${ }^{1}$. Com essa intervenção, busca-se desenvolvimento biopsicossocial de pessoas portadoras de necessidades especiais, trabalhando de forma interdisciplinar nas áreas de equitação, saúde e educação ${ }^{1,2}$.

A equoterapia é indicada para crianças e adultos em diversos casos, entre eles: paralisia cerebral, acidente vascular cerebral, trauma crânio encefálico, lesões medulares, síndromes, autismo, transtorno de déficit de atenção e hiperatividade, deficiência visual, deficiência auditiva, fobias, estresse e síndrome de Down ${ }^{1,2}$.

A equoterapia se inicia desde o primeiro contato entre praticante e animal. 0 praticante deve aprender a se aproximar do animal, interagir e montar; bem como criar estratégias para que o cavalo aceite seus comandos. Esse processo contribui para o desenvolvimento da afetividade e da confiança, além de colaborar para a aquisição de limites e respeito, nos quais o praticante tem responsabilidade perante o animal e deve cumprir algumas regras para o bom funcionamento do tratamento ${ }^{2}$.

No ambiente equoterápico, o praticante é o centro das atenções, no entanto, os ganhos conseguidos requerem esforço e paciência não só daqueles que recebem o tratamento, mas também de seus familiares ${ }^{3}$. Dadas as indicações, seus praticantes possuem níveis variados de dependência funcional e demandam auxílio de um cuidador em seu cotidiano. É frequente que os pais sejam os cuidadores dos praticantes de equoterapia, e que precisem aprender exercícios que serão realizados em casa, formas de aplicação de medicamentos, cuidados básicos de higiene, alimentação e sobre necessidades de adaptação do espaço físico dividido com o praticante ${ }^{2,3}$.

Apesar do importante papel desempenhado pelos cuidadores familiares, há poucos programas de apoio a tais cuidadores no Brasil ${ }^{4}$. Estudos ${ }^{4-7}$ indicam a existência de poucas propostas de intervenção para auxiliar cuidadores familiares de pessoas com quaisquer patologias, em descumprimento com as resoluções sobre saúde mental no Brasil e que indicam que os serviços não conseguem atender as demandas de orientação e auxílio dos familiares cuidadores.

Especificamente na equoterapia, não há intervenções pensadas para os cuidadores e as orientações dadas aos familiares são focadas nos praticantes. 0 apoio efetivo aos cuidadores familiares pode auxiliá-los a manter sua qualidade de vida ${ }^{3,6}$, minimizar a sobrecarga e outras questões emocionais que podem surgir como decorrência do papel de cuidadores, além de auxiliá-los a manter bons sentimentos sobre poder cuidar de um ente querido ${ }^{8,9}$.

Nesse sentido, propostas extensionistas podem representar uma importante ferramenta de modificação da realidade, aproximando o conhecimento universitário da comunidade e contribuindo para suprir lacunas de atuação junto aos cuidadores. Considerando essa realidade, o objetivo desse trabalho é relatar a experiência de um projeto de extensão baseado em intervenção psicológica breve realizado com cuidadores de praticantes de equoterapia.

\section{MÉTODO}

0 presente trabalho é um relato de experiência derivado de um projeto de extensão universitária desenvolvido com familiares cuidadores de praticantes de equoterapia que frequentavam a Associação Mineira de Equoterapia (AME), na cidade de Uberaba - MG, ao longo de 2018.

A AME é uma instituição filantrópica, fundada em 1998 para atender pessoas com diversas necessidades especiais. É composta por uma equipe multiprofissional das áreas de Fisioterapia, Psicologia, Terapia Ocupacional e Equitação. A instituição atendia, quando o trabalho foi conduzido, 71 praticantes cadastrados. Destes, 67 eram continuamente 
acompanhados por cuidadores familiares ou profissionais. Todos os cuidadores familiares foram convidados para o projeto de extensão.

0 projeto de extensão foi registrado na Universidade Federal do Triângulo Mineiro (UFTM) sob documento SIGPROJ 112391.480.29341.14042012). O projeto também contava com a autorização da instituição em que foi realizado e todos os participantes receberam explicações sobre o trabalho e assinaram o Termo de Consentimento Livre e Esclarecido, permitindo que seus resultados fossem comunicados em eventos e trabalhos científicos.

0 trabalho foi desenvolvido por três estudantes do curso de graduação em Psicologia da UFTM, supervisionados por uma psicóloga docente e pelo psicólogo da AME. Essa equipe convidou todos os familiares cuidadores que frequentavam a instituição no período de desenvolvimento do projeto. Havia um roteiro previamente elaborado para cada encontro, com base no referencial de atendimento breve, em abordagem cognitivo-comportamental e de temas indicados pelo setor de Psicologia da AME. As propostas de cada encontro foram sumarizados no Quadro 1.

Quadro 1. Roteiro dos encontros com os familiares. Uberaba, 2018.

\begin{tabular}{|c|c|c|}
\hline Encontro & Proposta & Agenda \\
\hline $1^{0}$ & $\begin{array}{l}\text { Estabelecimento } \\
\text { de vínculo e } \\
\text { levantamento de } \\
\text { informações }\end{array}$ & $\begin{array}{l}\text { - Explicar a natureza do projeto; } \\
\text { - Escuta empática sobre aspectos da experiência do cuidador, } \\
\text { identificando focos interventivos e temáticas de interesse individual. }\end{array}$ \\
\hline $2^{\circ}$ & $\begin{array}{l}\text { Levantamento de } \\
\text { demandas }\end{array}$ & $\begin{array}{l}\text { - Aplicação das escalas de avaliação de estado emocional, com } \\
\text { priorização da escala de sobrecarga } \\
\text { - Rastreio sobre desinformação sobre a patologia da pessoa sob } \\
\text { cuidados, possibilidades de tratamento e manejo de crises; } \\
\text { - Rastreio sobre as principais dificuldades como cuidadores; } \\
\text { - Rastreio de potencialidades não incentivadas do paciente. }\end{array}$ \\
\hline 30 & $\begin{array}{l}\text { Estabelecimento } \\
\text { de metas }\end{array}$ & $\begin{array}{l}\text { - Definição de metas para autocuidado; } \\
\text { - Definição de metas como cuidador } \\
\text { - Esclarecimentos sobre a patologia do praticante e outros pontos } \\
\text { levantados no encontro anterior. }\end{array}$ \\
\hline $4^{\circ}$ ao $6^{0}$ & Planificação & $\begin{array}{l}\text { - Levantamento de possibilidades concretas de reestruturação de } \\
\text { rotina e atividades de autocuidado; } \\
\text { - Trabalhar sentimentos de culpa na relação cuidador x autocuidado; } \\
\text { - Levantamento de possibilidades de cessão de atividades de cuidado } \\
\text { ou formas mais brandas de monitoramento do praticante por parte do } \\
\text { cuidador. }\end{array}$ \\
\hline 79 & Planificação & $\begin{array}{l}\text { - Ensinar técnica de relaxamento físico progressivo e respiração } \\
\text { diafragmática; } \\
\text { - Trabalhar a importância das "válvulas de escape" e preparação para } \\
\text { perda na vida dos cuidadores; } \\
\text { Atividade prevista para casa: Treinamento do relaxamento físico. }\end{array}$ \\
\hline $8^{\circ}$ & $\begin{array}{l}\text { Desenvolvimento } \\
\text { de tema } \\
\text { individuais }\end{array}$ & $\begin{array}{l}\text { - Encontro destinado ao desenvolvimento de temas anteriores de } \\
\text { agenda que precisam ser melhor trabalhados e propostas específicas } \\
\text { para a realidade do cuidador; } \\
\text { Verificação do aprendizado do relaxamento físico. }\end{array}$ \\
\hline 9o & Encerramento & $\begin{array}{l}\text { - Devolução das características observadas nas escalas; } \\
\text { Retomada dos temas trabalhados; } \\
\text { Feedback sobre o desenvolvimento do trabalho. }\end{array}$ \\
\hline
\end{tabular}

Os familiares eram abordados individualmente enquanto aguardavam a realização da sessão de equoterapia da pessoa a quem prestavam cuidados. A organização do trabalho foi feita em forma de nove encontros (8 temáticos e 1 de fechamento/feedback), baseados em conversas individuais ou com os casais (segundo preferência dos participantes), com limite máximo de tempo de 50 minutos cada, que era o tempo de duração das sessões de equoterapia.

Ao longo dos encontros, a rotina e os hobbies dos cuidadores foram identificados e sistematizados, e as temáticas previstas foram adaptadas, quando necessário. A equipe utilizou 
de escuta ativa não punitiva, uma técnica da abordagem cognitivo-comportamental ${ }^{10}$, para buscar compreender as histórias de vida dos familiares, bem como sua rotina de vida antes e após se tornarem cuidadores, suas expectativas pessoais, e os participantes foram incentivados e auxiliados a identificar suas necessidades pessoais.

Ao término de cada encontro, os estudantes faziam anotações sobre os temas trabalhados, os sentimentos relatados pelos familiares e os próprios sentimentos por vivenciar a experiência de conversar com os cuidadores. Essas anotações eram levadas para os encontros de supervisão e faziam parte do processo de autoavaliação contínua da proposta.

\section{RESULTADOS}

Participaram do projeto 19 cuidadores familiares (5 homens e 14 mulheres), com idades entre 26 e 63 anos. Tais cuidadores eram pais, padrastos ou mães dos praticantes e cuidavam de pessoas com diagnóstico de paralisia cerebral, autismo ou síndrome de Down.

As conversas com os cuidadores mostraram que as mães tinham mais relatos de sobrecarga e indicavam uma rotina de cuidados muito mais intensa do que os pais. Em geral, os pais trabalhavam fora em horário integral e auxiliavam os praticantes nos cuidados prestados no período da noite, em tarefas como tomar banho e se preparar para dormir. Algumas mães possuíam empregos de tempo integral, mas conciliavam a tarefa de cuidar com as demandas de trabalho. As demandas das tarefas de cuidado e possibilidades de divisão dessas atividades com outros familiares foram discutidas com os cuidadores e algumas metas individuais foram construídas ao longo dos encontros.

Outro ponto trabalhado com os familiares foi a possível relação entre sobrecarga, sintomas emocionais negativos (de depressão e estresse) e como esses aspectos podiam prejudicar seu autocuidado. Muitos familiares relataram crenças disfuncionais sobre não conseguirem ser bons cuidadores, terem abdicado de suas vidas e se ressentirem da pessoa de quem cuidavam por causa disso, entre outras.

Os familiares que mostraram esse tipo de fala responderam a uma escala de sintomas depressivos e foram triados para depressão em graus entre moderado e severo, mas nenhum desses cuidadores havia procurado por acompanhamento psicológico/psiquiátrico. Assim, durante as conversas com tais cuidadores, esse foi outro ponto trabalhado e foram feitos dois encaminhamentos para serviços de saúde da cidade, para avaliação de possível diagnóstico de depressão.

Ao analisar, juntamente com os familiares, as atividades que realizavam antes de se tornarem cuidadores e as que mantinham no momento da intervenção foi possível perceber que os cuidadores abdicaram de muitas atividades cotidianas individuais, referentes ao trabalho ou lazer e alegavam ter precisado fazer isso para cuidar dos praticantes.

A rotina familiar passou a ser montada em torno das necessidades do praticante. Os cuidadores não localizavam tempo ou valorizavam atividades como ouvir música, fazer uma refeição sem pressa ou descansar, mesmo cuidadores que realizavam atividades de lazer antes de assumirem essa função abandonaram tais práticas para conseguir tempo para realização das atividades de cuidado (levar a pessoa que cuidavam para tratamento de saúde, para escola e atividades extracurriculares) e sociais (ir à praça ou ao aniversário de outras crianças).

A partir da construção da rotina do cuidador, foi observado que eram raros os momentos em que os cuidadores faziam algo focado em seu próprio bem-estar ou autocuidado, e tinham dificuldade para encontrar outras pessoas para dividir as responsabilidades pelo cuidado. As crenças disfuncionais sobre a falta de valorização do autocuidado e de tempo para as atividades de lazer foi objeto de intervenção.

Ao discutir juntamente com os familiares suas possibilidades de adaptação na rotina, e necessidade de valorização do autocuidado, percebeu-se o engessamento de crenças sobre a incapacidade de realizar essas ações e a resistência em tentar realizar alterações na rotina familiar. Ao longo dos encontros e avanço das discussões, alguns cuidadores mostraram 
espanto ao conseguir fazer mudanças em seu cotidiano para incluir cursos de artesanato ou atividades de autocuidado (caminhada, procurar tratamento para questões próprias de saúde, ir ao salão de beleza fazer as unhas) e conseguir realizar as atividades de cuidado.

Nos espaços de diálogo foi possível perceber que os cuidadores não se queixavam tanto das atividades que precisavam realizar, mas das relações pessoais. Houve relatos sobre críticas recebidas do marido/esposa, interferências de outros familiares na dinâmica nuclear da família, falhas na comunicação conjugal, problemas relacionados ao serviço doméstico e com o desempenho de funcionárias contratadas para auxiliar em casa e a forma como se sentiam por se perceberem excluídos do planejamento familiar, não reconhecidos e desvalorizados, apesar de sua dedicação.

0 desenrolar dos encontros permitiu perceber o impacto da vivência de cuidadores na dinâmica familiar, inclusive na família extensa. Os relatos falavam de falta de apoio para cuidar, distanciamento entre o casal, culpabilização de um dos pais pela patologia do praticante por parte da família extensa, falta de diálogo e ausência de atividades feitas em família.

O desconhecimento sobre as patologias e a falta de preparação dos ambientes para lidar com crianças com necessidades especiais apareceu como outro dificultador relatado pelos cuidadores de praticantes de equoterapia. Esse despreparo os obrigava a responder perguntas recorrentes sobre as patologias dos praticantes, lidar com comentários errôneos ou depreciativos sobre as pessoas de quem cuidam, e lidar com preconceito, aberto ou velado. Houve relatos sobre os filhos não receberem convites para festas de outras crianças, sobre dificuldades para frequentar restaurantes porque as pessoas olhavam de forma estranha, ouvir comentários denotativos de piedade pelo praticante ser "inválido", entre outros comentários sociais que geravam sofrimento para os cuidadores.

As conversas com os cuidadores também mostraram desinformação mesmo entre os cuidadores. Alguns pais não permitiam que os praticantes realizassem atividades possíveis dentro de casa, tais como retirar biscoitos de um pote para comer ou dormir sozinhos em seus quartos, por medo de que engasgassem. Outros não acreditavam que a pessoa de que cuidavam seriam totalmente dependentes. Esses foram aspectos trabalhados ao longo dos encontros, mostrando aos pais casos públicos de superação de limitações físicas ou intelectuais e o papel dos apoiadores no desenvolvimento da independência funcional e sendo de competência. Esses foram aspectos largamente destacados nas devolutivas com os familiares e a instituição.

De modo geral, os cuidadores que participaram do projeto de extensão forneceram, no momento de feedback sobre o trabalho, que perceberam diferenças em seus sentimentos e suas rotinas ao longo da realização dos encontros. Alguns indicaram que negociaram uma melhor divisão de tarefas com outros familiares e incluíram atividades de lazer na programação semanal da família e relataram a intenção de manter essas mudanças no futuro, por perceber seu impacto positivo na forma como se sentiam e no funcionamento familiar, sem prejudicar os cuidados prestados. Não houve relatos negativos durante os feedbacks.

Os familiares relataram, ainda, que gostariam que propostas como essa fossem mantidas na instituição, pois interpretaram que estas os ajudaram a problematizar e organizar pontos em que tinham dificuldades.

\section{DISCUSSÃO}

Ao longo dos encontros percebeu-se que familiares cuidadores de praticantes de equoterapia eram, predominantemente, as mães dos participantes. Esse perfil corrobora o identificado anteriormente em estudos com cuidadores de pessoas com diversas patologias ${ }^{3,4}$. A maioria das participantes relatou ter precisado abdicar do emprego para poder se dedicar integralmente ao cuidado.

Alguns estudos ${ }^{1,6,9}$ indicam que é comum que os cuidadores familiares remodelem sua rotina de vida em benefício das pessoas de quem cuidam, assumindo compromissos e horários rígidos. Essa situação se agrava quando a função de cuidadores foi assumida há menos tempo, 
por não existir treinamento ou cursos de preparação prévia, o que pode levar os cuidadores a abrir mão primeiro de atividades de cunho individual, que seriam essenciais para manutenção de sua saúde física e mental ${ }^{8}$.

Além disso, seguindo a tendência observada em outros trabalhos ${ }^{5,7,8}$, observou-se presença de sobrecarga e estresse na maioria dos cuidadores. Houve também identificação de sintomas depressivos em vários cuidadores. As tarefas de cuidar serem responsabilidade feminina e as cuidadoras precisarem abdicar de atividades próprias para prover o cuidador faz parte do perfil anteriormente identificado em mulheres cuidadoras ${ }^{3,4}$. Esses achados mostram um papel social atribuído as mulheres e uma fonte importante da sobrecarga relatada pelas cuidadoras.

A vivência de sobrecarga é um achado frequente nos trabalhos com cuidadores ${ }^{5,8,9}$ e pode se relacionar com o surgimento de outros quadros emocionais negativos, como estresse e depressão ${ }^{11}$. Esses aspectos negativos somam-se às pressões sociais e tornam a vivência como cuidador um fator de risco para a qualidade de vida dessa população e para a qualidade dos cuidados que prestam a outro(s) ${ }^{9}$.

É mais frequente encontrar pesquisas que avaliam o estado emocional dos cuidadores do que propostas de intervenção para auxiliarem tais pessoas ${ }^{6,9}$. Ao longo do presente trabalho a organização dos encontros se pautou na lógica da intervenção psicológica breve com referencial cognitivo-comportamental ${ }^{10}$, mostrando a possibilidade que trabalhos similares sejam bem recebidos pelos cuidadores e tenham impacto positivo na vida dos cuidadores.

Tais trabalhos podem servir como momento de escuta, valorização da condição de pessoa, além do reconhecimento do trabalho como cuidador e, ainda, auxiliar na identificação e encaminhamento precoce ${ }^{10}$. Essa possibilidade se mostrou valiosa quando foram retomados aspectos de saúde com os cuidadores e fez-se o encaminhamento de alguns deles, triados para depressão, para buscar ajuda profissional. Na devolutiva feita à instituição, após o encerramento do projeto, foi feita a indicação da criação de um serviço de acompanhamento aos familiares cuidadores ou da montagem de uma rede de parceria com profissionais que pudessem ocupar esse lugar, caso a AME não dispusesse de condições para fazê-lo.

0 principal ponto de sofrimento destacado pelos cuidadores não foi a quantidade de trabalho para prover cuidados e sim aspectos subjetivos, como não se sentirem respeitados e reconhecidos. Essas observações corroboram com os estudos sobre a sobrecarga ${ }^{8}$, que destacam que os cuidadores podem sentir de forma diferente o desgaste por ter que desempenhar atividades concretas de cuidado (sobrecarga objetiva) e a forma que se sentem por precisar fazer isso (sobrecarga subjetiva). Assim, intervenções que foquem nos cuidadores de pessoas que praticam equoterapia precisam incluir tanto momentos para auxiliar tais cuidadores na organização de suas rotinas e divisão objetiva de tarefas de forma a preservar momentos de autocuidado e lazer, quanto auxiliá-los no processo

\section{CONCLUSÃO}

0 trabalho realizado demonstrou o quanto os cuidadores organizam suas vidas em função das atividades das pessoas a quem prestam cuidados, muitas vezes anulando suas próprias necessidades. Na proposta de intervenção relatada, optou-se por tentar demonstrar, por meio de conversas e do levantamento de seus gostos, rotina e sentimentos, a necessidade do autocuidado.

Foram observados bons resultados dessa abordagem e recebidos feedbacks dos cuidadores de praticantes de equoterapia que perceberam-se beneficiados com o trabalho. Essa percepção ilustra a importância das instituições considerarem as demandas dos familiares cuidadores e, caso não tenham condições de prover tal cuidado, reitera-se a importância que indiquem sua importância e ajudem os familiares a buscar por locais em que podem ter suas necessidades atendidas. 
Há algumas limitações que precisam ser indicadas. 0 projeto foi realizado ao longo de dois semestres e não pôde ser continuado, devido a outros compromissos profissionais da equipe que o conduziu. Além disso, como os horários eram restritos, nem todos os cuidadores de praticantes de equoterapia tiveram possibilidade de participar.

Apesar de tais limitações, houve ganhos com a experiência. A abertura da AME para esse trabalho mostrou-se importante para os familiares que participaram do projeto de extensão e para tornar visível a carência de intervenções com esse foco. Essa oportunidade mostrou, ainda, que é possível desenvolver trabalhos de intervenção de baixo custo e rápida execução, capazes de gerar impactos positivos na vida dos cuidadores, podendo esse estudo servir de base para trabalhos futuros com outros cuidadores.

\section{REFERÊNCIAS}

1. Souza VM, Aquino GB, Silva AO. Psicologia e equoterapia: conhecendo as dificuldades enfrentadas pelos cuidadores dos praticantes. Rev Cient Faminas [Internet]. 2011 [citado em 10 nov 2020]; 7(3):125-40. Disponível em: http://periodicos.faminas.edu.br/index.php/RCFaminas/article/view/284

2. Silva JP, Aguiar OX. Equoterapia em crianças com necessidades especiais. Rev Cient Eletr Psicol. [Internet]. 2008 [citado em 20 out 2020]; 6(11):12-5. Disponível em: http://faef.revista.inf.br/imagens_arquivos/arquivos_destaque/pMX6nTKTbW28ch4_20135-13-12-35-25.pdf

3. Vieira MLN. Participação dos cuidadores na equoterapia In: Soares DFG, Pinto EF, Otone GA, Silveira MAS, Carvalho R, organizadores. Equoterapia: teoria e prática no Brasil. Uberaba: FUNEC; 2013. p. 541-554.

4. Barroso SM, Silva LF. Cuidadores informais e profissionais. In: Barroso SM, organizadora. Desafios (in)visíveis dos cuidadores familiares e profissionais. Rio de Janeiro: Editora Autografia; 2017. p. 12-28.

5. Machado BM, Dahdah DF, Kebbe LM. Caregivers of family members with chronic diseases: coping strategies used in everyday life. Cad Bras Ter Ocup. [Internet]. 2018 [citado em 20 nov 2020]; 26(2):299-313. DOI: http://dx.doi.org/10.4322/2526-8910.ctoao1188

6. Barroso SM, Bandeira M. Os serviços psiquiátricos estão atendendo às necessidades dos familiares cuidadores? In: Barroso SM, Scorsolini-Comin F, organizadores. Diálogos em psicologia: práticas profissionais e produção do conhecimento. Uberaba, MG: Editora UFTM; 2012. p. 123-36.

7. Sorato DB, Peres SV, Mitsuyuki MC, Drude FS. Cuidar e ser cuidado pelo grupo de apoio PROTEGE. Psicol Estud. [Internet]. 2010 [citado em 05 nov 2020]; 15(4):751-9. DOI: https://doi.org/10.1590/S1413-73722010000400011

8. Fiorotto SM. Resiliência e sobrecarga: um estudo sobre cuidadores de pacientes com Alzheimer. In: Barroso SM, organizadora. Desafios (in)visíveis dos cuidadores familiares e profissionais. Rio de Janeiro: Editora Autografia; 2017. p. 103-19.

9. Maffioletti VLR, Baptista MAT, Santos RL, Rodrigues VM, Dourado MCN. Effectiveness of day care in supporting family caregivers of people with dementia: a systematic review. Dement Neuropsychol. [Internet]. 2019 [citado em 20 nov 2020]; 13(3):268-83. DOI: https://doi.org/10.1590/1980-57642018dn13-030003

10. García-Cardoza II, Zapata-Vázquez R, Rivas-Acuña V, Quevedo-Tejero EC. Efectos de la terapia cognitivo-conductual en la sobrecarga del cuidador primario de adultos mayores. Horiz Sanitario [Internet]. 2018 [citado em 20 nov 2020]; 17(2):131-40. DOI: http://doi.org/10.19136/hs.a17n2.2039

11. Duarte ESR, Silveira LVA, Cítero VA, Jacinto AF. Common mental disorder among family carers of demented older people in Brazil. Dement Neuropsychol. [Internet]. 2018 [citado em 20 nov 2020]; 12(4):402-7. DOI: https://doi.org/10.1590/1980-57642018dn12-040010 


\section{CONTRIBUIÇÕES}

Sabrina Martins Barroso atuou no desenho e orientação do estudo, redação e revisão. Juliana Machado Ruiz, Camila Aparecida Peres Borges e Dennis Gabiatti Lopes contribuíram no desenho do estudo, conduziram os encontros com os familiares e redação. Cleyton Magela Reis orientou o projeto e participou da revisão.

\section{Como citar este artigo (Vancouver)}

Barroso SM, Ruiz JM, Borges CAP, Reis CM, Lopes DG. Relato de experiência sobre uma intervenção psicológica com familiares de praticantes de equoterapia. REFACS [Internet]. 2021 [citado em inserir dia, mês e ano de acesso]; 9(1):151-158. Disponível em: inserir link de acesso. DOI: inserir link do DOI

\section{Como citar este artigo (ABNT)}

BARROSO, S. M.; RUIZ, J. M.; BORGES, C. A. P.; REIS, C. M.; LOPES, D. G. Relato de experiência sobre uma intervenção psicológica com familiares de praticantes de equoterapia. REFACS, Uberaba, MG, v. 9, n. 1, p. 151-158, 2021. DOI: inserir link do DOI. Disponível em: inserir link de acesso. Acesso em: inserir dia, mês e ano de acesso.

\section{Como citar este artigo (APA)}

Barroso, S.M., Ruiz, J.M., Borges, C.A.P., Reis, C.M., \& Lopes, D.G. (2021). Relato de experiência sobre uma intervenção psicológica com familiares de praticantes de equoterapia. REFACS, 9(1), 151-158. Recuperado em inserir dia, mês e ano de acesso de inserir link de acesso. DOI: inserir link do DOI. 